\title{
Experiencia académica y comprensión de textos especializados en estudiantes universitarios de Ciencias Políticas*
}

\author{
Academic experience and specialized text comprehension of college \\ students in Political Science

\begin{abstract}
Acadêmico experiência e compreensão dos textos especializados dos estudantes universitários em Ciência Política
\end{abstract}

\author{
Bernardo Riffo Ocares, ${ }^{\mathrm{a}}$ Marco Contreras Castro $^{\mathrm{b}}$ \\ aUniversidad de Concepción, Chile. Correo electrónico: bernardo@udec.cl \\ bUniversidad de Concepción, Chile. Correo electrónico: marcontrerasc@udec.cl
}

\begin{abstract}
RESUMEN
En el presente artículo se da cuenta de los resultados de una investigación descriptiva de la comprensión de textos especializados en estudiantes universitarios de la carrera de Ciencias Políticas. La hipótesis de trabajo postula que tanto el nivel de formación académica como el grado de especialización de los textos, inciden significativamente en el rendimiento de los lectores. Se elaboraron dos pruebas de comprensión lectora con textos especializados propuestos por los profesores de la carrera. Para cada texto se formularon preguntas con el propósito de medir diversos aspectos de la lectura. El instrumento fue aplicado a una población de 55 individuos correspondientes a tres niveles de formación profesional y académica. Los resultados permiten confirmar la hipótesis y, al mismo tiempo, identificar una serie de aspectos que requieren el desarrollo de futuras investigaciones más específicas.
\end{abstract}

Palabras clave: comprensión de lectura, textos especializados.

\begin{abstract}
This article presents the results of a descriptive study of specialized texts comprehension in college students of Political Science. The working hypothesis postulates that both the academic level and the specialization degree of these texts have a significant impact on the performance of readers. Two specialized texts reading tests were developed. The texts were proposed by the experts. For each text there were questions in order to measure various aspects of reading. The instrument was applied to a population of 55 individuals for three levels of academic training. The results confirmed the hypothesis and, at the same time, allowed to identify a number of issues requiring the development of further more specific research.
\end{abstract}

Key words: reading comprehension, specialized texts.

\section{RESUMO}

Neste artigo vamos apresentar os resultados de um estudo descritivo de compreensão de leitura em alunos universitários especializados na carreira de Ciência Política. A hipótese de trabalho postula que o nível de formação acadêmica e ao grau de especialização dos textos impacto significativo sobre o desempenho dos leitores. Ela se desenvolveu dois testes na leitura de textos especializados propostas pelos professores da corrida. Para cada texto havia perguntas a fim de medir os diferentes aspectos da leitura. $\mathrm{O}$ instrumento foi aplicado a uma população de 55 indivíduos por três níveis de formação profissional e acadêmica. Os resultados confirmam a hipótese e, ao mesmo tempo, identificar uma série de questões que exigem mais pesquisas para desenvolver mais específico.

Palavras chave: compreensão de leitura, textos especializados. 


\section{INTRODUCCIÓN}

El desarrollo de habilidades complejas como aquellas que se requieren para leer comprensivamente un texto constituye un proceso en el que confluyen factores de diversa índole. La psicolingüística ha propuesto teorías ampliamente conocidas (van Dijk \& Kintsch, 1983; Gernsbacher, 1997; Kintsch, 1998; Zwan \& Radvansky, 1998; entre otros) que dan cuenta tanto de los mecanismos que hacen posible la elaboración de representaciones mentales vinculadas a la lectura, como de la naturaleza de las mismas y su lugar en la memoria. Ha sido foco de interés para esta disciplina el desarrollo de modelos explicativos centrados en la dimensión cognitiva, especialmente en los aspectos procedimentales correspondientes a la operatoria de la comprensión. Si bien el texto mismo como un componente del fenómeno observado ha formado parte de las propuestas antes mencionadas, su importancia no ha alcanzado el mismo status que otros aspectos como el conocimiento previo o los procesos inferenciales necesarios para la comprensión. La lectura de textos especializados, a su vez, constituye una problemática solo de reciente interés para la psicolingüística (Parodi, 2005). Por otra parte, los modelos teóricos han supuesto un lector relativamente estable en sus capacidades y con un cierto grado de competencia ya logrado. Por el contrario, el proceso evolutivo que supone cambios en la competencia lectora ha recibido menos atención o bien no forma parte explícita de las propuestas teóricas. En este estudio nos proponemos dar cuenta de este aspecto mediante un estudio descriptivo basado en los resultados de dos pruebas de comprensión lectora aplicadas a estudiantes universitarios de Ciencias Políticas situados en tres etapas distintas de su formación. Con ello, nos propusimos averiguar en qué medida el nivel de formación alcanzado por los estudiantes y el grado de especialización de los textos inciden en el rendimiento lector.

\subsection{LA COMPRENSIÓN DEL DISCURSO}

En lo fundamental, los distintos modelos teóricos de la comprensión lectora coinciden en que el proceso consiste básicamente en la decodificación de la señal lingüística, su procesamiento en diversos niveles de la estructura del código lingüístico (fonológico, léxico, sintáctico, textual) y su vinculación con la situación comunicativa (dimensión pragmática). Existe hoy un cierto consenso en admitir que cada nivel de procesamiento implica la elaboración de representaciones mentales específicas producto de operaciones que se rigen por estrategias o modos de ejecución propios de cada componente lingüístico. Estas representaciones se integran finalmente en una de mayor envergadura, complejidad e importancia, a saber, el modelo de situación o modelo mental (van Dijk \& Kintsch, 1983; Johnson-Laird, 1983; Kintsch, 1998; Zwaan \& Radvansky, 1998). La comprensión cabal de un texto requiere de un modelo en la forma de una representación cognitiva no proposicional y análoga, la que se entiende necesaria, entre otras funciones, para el razonamiento y la realización de inferencias. En términos más simples, comprender un texto implica entender "de qué trata" el mismo; sólo de este modo es posible luego hacer uso de la información obtenida.

Para alcanzar el objetivo, es decir, la construcción del modelo de situación, el lector debe recurrir a su conocimiento del lenguaje, del tema tratado y al conocimiento procedimental sobre la operatoria de la lectura. Todo lo anterior supone un conjunto 
de competencias adquiridas en múltiples experiencias vividas en diferentes contextos a través de un extenso proceso evolutivo que, en el caso de lectores adultos que leen textos especializados en ambientes académicos, se extiende por muchos años. En efecto, alcanzar una capacidad de leer artículos científicos, manuales, informes de laboratorio y textos teóricos de una disciplina científica supone, primero, que se han alcanzado otras habilidades de lectura, unas más básicas y de adquisición más temprana, por cierto. Un estudiante universitario lo es en la medida que puede demostrar, en los procedimientos de selección para el ingreso a una universidad, ciertas competencias mínimas en el dominio de la lengua formal culta. Estas capacidades suponen, a su vez, otras habilidades previas relacionadas con la mecánica de la lectura en los niveles más elementales de la organización del texto. La lectura de textos académicos se presenta así como un desempeño intelectual de la más alta complejidad. En consecuencia, no es algo que se desarrolle en forma espontánea; sino que, por el contrario, ocurre solo en el marco de un espacio de aprendizaje sistemático y con objetivos explícitos.

\subsection{LA LECTURA DE TEXTOS ESPECIALIZADOS}

Como bien señala Parodi (2005), en un mundo tan dinámico y complejo en sus mecanismos de construcción y transmisión de conocimiento, el discurso especializado constituye un pilar fundamental para los procesos educativos tendientes a formar nuevos profesionales en distintas disciplinas. En este contexto, el desarrollo de habilidades para la lectura comprensiva de textos especializados constituye hoy un punto gravitante en el desarrollo de los sistemas de enseñanza en la educación terciaria, toda vez que el lenguaje escrito es medio preferente para la creación, fijación y transmisión del conocimiento disciplinar (Parodi, 2008). El ingreso de los jóvenes a la universidad puede ser visto como un proceso de aculturación en tanto conlleva su incorporación a una comunidad discursiva específica que alberga una cultura académica por completo nueva para el joven estudiante. Dicha comunidad se caracteriza, entre otros rasgos, por el dominio de ciertos sistemas de comunicación presentes entre sus miembros, los que incluyen textos disciplinares de variada especificidad que van desde los más didácticos (con finalidad formativa), hasta los más especializados y restringidos. Tales propiedades del discurso disciplinar permiten predecir que, según la complejidad y grado de especialización de los mismos, estos presentarán diversos niveles de lecturabilidad, esto es, que los diversos textos representarán diferentes niveles de dificultad para los nuevos lectores dependiendo de su complejidad (determinada en gran parte por su especialización). Por otra parte, la dificultad intrínseca de los textos se explica en relación con las habilidades del lector. El experto y el neófito no leerán de igual forma un mismo texto.

La importancia de la lectura con propósitos académicos radica en que este proceso forma parte de la práctica social de transmisión de conocimiento dentro de una entidad altamente institucionalizada y convencionalizada, que crea restricciones y requisitos, y que demanda de los lectores la apropiación de las convenciones que regulan la comunidad (Carlino, 2005). Desenvolverse en estos ambientes de aprendizaje con un lenguaje especializado implica adquirir unos conocimientos lingüísticos específicos, y las condiciones bajo las cuales la comunidad discursiva los utiliza y que afectan a todos los niveles de organización del lenguaje, es decir, la fonología, la gramática y el léxico, e incluyen también los aspectos socioculturales, pragmáticos y retóricos propios de cada 
práctica comunicativa: qué se comunica, cómo, de qué manera, para qué y con quién (Cassany, 2008).

Considerados estos antecedentes, nos hemos propuesto realizar un estudio para averiguar en qué medida el nivel de formación académica y la complejidad de los textos especializados inciden en el desempeño lector de estudiantes universitarios. La primera variable representa, en cierta medida, el grado de incorporación a la comunidad discursiva especializada, ya que el número de años de estudio supone diversos niveles de conocimiento no solo de la disciplina, sino también de las formas lingüísticas (géneros discursivos) propias de la ciencia. La segunda variable, a su vez, debería representar un determinado nivel de dificultad intrínseco, ya que el mayor o menor grado de especialización de un texto lo hará más fácil o difícil de comprender para un estudiante.

\section{LA INVESTIGACIÓN}

\subsection{OBJETIVOS}

a) Determinar si el nivel de formación académica incide en el desempeño de los sujetos cuando leen textos especializados.

b) Determinar si el grado de especialización del texto incide en el desempeño de los sujetos.

\subsection{HIPÓTESIS}

El nivel de formación académica y el grado de especialización del discurso inciden significativamente en el desempeño de estudiantes universitarios de Ciencias Políticas en pruebas de comprensión de textos disciplinares.

\subsection{DISEÑO}

Con el propósito de someter a prueba la hipótesis postulada, se llevó a efecto el estudio empírico. El diseño escogido fue de tipo descriptivo, consistente en dos pruebas de comprensión lectora aplicadas en condiciones habituales de trabajo académico de los sujetos participantes, pruebas que incluían la lectura de dos textos especializados, respecto de los cuales los estudiantes debían responder una serie de preguntas. Los resultados fueron cuantificados y analizados según se detalla más adelante.

\subsection{PROCEDIMIENTOS}

a) Población participante. Esta estuvo conformada por 55 estudiantes universitarios de la carrera de Ciencias Políticas de la Universidad de Concepción, distribuidos en tres grupos: 20 estudiantes de primer semestre (primer año), 20 de quinto semestre (tercer año) y 15 del noveno semestre (quinto año). Los alumnos fueron reclutados en el marco de actividades académicas regulares establecidas por la carrera y en la que debían participar todos los estudiantes convocados.

b) Las pruebas. Este procedimiento incluyó dos momentos: primero, la selección de los textos y, segundo, la confección de las pruebas que consideraba la elaboración de 
un cuestionario para cada texto. En el primer caso, decidimos no optar por un análisis lingüístico estructural para determinar el grado de especialización de los textos (variable que debía ser controlada, toda vez que forma parte del problema científico y su hipótesis respectiva); por el contrario, se consultó a los integrantes de la propia comunidad discursiva solicitándoles proporcionaran material discursivo que se considera específico de la disciplina. Se instruyó a los académicos a fin de que los textos seleccionados cumplieran con los requisitos que ellos consideraban característicos de cada género discursivo. Se les solicitó, además, que diferenciaran entre textos con distinto grado de especialización, en otras palabras, que distinguieran entre textos que requerían mayor formación disciplinar y aquellos más accesibles para un lector menos especializado. En cumplimiento con lo anterior, se elaboró una encuesta para los docentes de la carrera, quienes cooperaron activamente en el proyecto, fruto de lo cual se obtuvo un conjunto considerable de materiales, de entre los cuales se seleccionaron dos textos de acuerdo con el juicio de los expertos en términos de sus propiedades, vale decir, uno de alta especialización y otro de carácter más didáctico y de menor exigencia académica específica (ver copia de los textos en anexo). En el segundo caso, una vez obtenidos los dos textos, se elaboró un cuestionario consistente en una extensa lista de preguntas abiertas siguiendo los criterios de Véliz \& Riffo (1993). Estos autores elaboraron una propuesta para la evaluación de la comprensión lectora basada en el modelo estratégico de van Dijk y Kintsch (1983). Básicamente, los criterios distinguen niveles de comprensión rotulados como nivel proposicional (comprensión de una proposición), nivel local (comprensión de la microestructura conformada por dos proposiciones relacionadas) y nivel global (comprensión de la macroestructura). Dentro de cada nivel se distinguen diversos tipos de relación entre los elementos, las que pueden ser de carácter condicional o funcional. Al mismo tiempo, se hace una distinción entre la información explícita e implícita en el texto, lo que da lugar a las preguntas de respuesta explícita (PRE) y las de respuesta implícita (PRI). Hemos agregado en esta investigación un nivel que denominamos "elaborativo", que corresponde a las tareas de comprensión que requieren unas inferencias de mayor complejidad y que ocurren cuando la respuesta exige construir o elaborar (de ahí su nombre) la información uniendo elementos de diversas fuentes. Cabe señalar aquí que las preguntas y su correspondiente pauta de corrección fueron revisadas por los expertos, quienes sugirieron las modificaciones que en su opinión debían introducirse. Producto de ello se obtuvo un total de 12 preguntas para cada texto, según se resume en la Tabla 1.

\begin{tabular}{|c|c|c|c|c|}
\hline \multicolumn{2}{|c|}{ Nivel } & $\begin{array}{c}\text { Cantidad de } \\
\text { preguntas }\end{array}$ & $\begin{array}{c}\text { Preguntas Prueba } \\
\text { I (número } \\
\text { correlativo) }\end{array}$ & $\begin{array}{l}\text { Preguntas Prueba II } \\
\text { (número correlativo) }\end{array}$ \\
\hline \multicolumn{2}{|c|}{ Proposicional (PRE) } & 2 & 4,6 & 1,6 \\
\hline \multirow{3}{*}{ Local } & $\begin{array}{l}\text { Funcional } \\
\text { (PRI) }\end{array}$ & 2 & 8,12 & 5,9 \\
\hline & $\begin{array}{l}\text { Condicional } \\
\text { (PRE) }\end{array}$ & 1 & 1 & 7 \\
\hline & $\begin{array}{l}\text { Condicional } \\
\text { (PRI) }\end{array}$ & 1 & 3 & 3 \\
\hline
\end{tabular}




\begin{tabular}{|c|c|c|c|c|}
\hline \multirow[t]{3}{*}{ Global } & $\begin{array}{c}\text { Idea Global } \\
\text { (PRI) }\end{array}$ & 1 & 2 & 8 \\
\hline & $\begin{array}{c}\text { Condicional } \\
\text { (PRE) }\end{array}$ & 1 & 11 & 12 \\
\hline & $\begin{array}{c}\text { Condicional } \\
(\mathrm{PRI})\end{array}$ & 2 & 5,9 & 10,11 \\
\hline Elaborativo & $\begin{array}{c}\text { Inferencial } \\
\text { Elaborativo } \\
\text { (PRI) }\end{array}$ & 2 & 7,10 & 2,4 \\
\hline \multicolumn{2}{|c|}{ TOTAL } & 12 & & \\
\hline
\end{tabular}

Tabla 1. Distribución de las preguntas según clasificación de Véliz \& Riffo (1993)

c) Aplicación de las pruebas. Luego de fijar tanto los textos como las preguntas y su pauta de corrección, se realizó una aplicación piloto a un número reducido de estudiantes de la carrera que no pertenecían a ninguna de las cohortes seleccionadas para la aplicación definitiva. Una vez efectuada la prueba piloto y analizados los resultados, se determinó realizar ajustes en la redacción de algunas preguntas y se estimó el tiempo requerido por los sujetos para responder las pruebas. Finalmente, se procedió a aplicar la versión final de las pruebas a los tres grupos participantes, cada uno de los cuales respondió la prueba en distintos momentos.

\section{RESULTADOS}

Los resultados que a continuación se presentan, corresponden al análisis cuantitativo de los puntajes obtenidos por los sujetos en las pruebas de comprensión. Cada pregunta tenía como máximo un valor de 2 puntos para una respuesta correcta. A una respuesta parcialmente correcta se le asignó 1 punto. Aquellas respuestas erróneas o en blanco recibieron 0 puntos. Para las pruebas estadísticas se utilizó el software Infosat (2009). Los resultados se expresan como promedios \pm desviación estándar. Las variables con distribución normal fueron analizadas mediante un estudio varianza (ANOVA) y las que no verificaron la normalidad se analizaron mediante el estudio de varianza no paramétrica de Kruskall Wallis. Cuando el valor p asociado a dichas pruebas resultó significativo, se procedió a realizar el test de comparaciones múltiples de Tukey. Se consideró un nivel de significancia del 0.05 (valor p de la prueba). Por último, se verificó la normalidad de las variables mediante la prueba de Shapiro-Wilk.

\subsection{RESULTADOS GLOBALES}

Los resultados globales resumidos en la Tabla 2 muestran una tendencia clara a favor de la hipótesis propuesta, esto es, a mayor nivel de formación académica, mayor rendimiento en las pruebas. En efecto, los tres grupos se diferencian significativamente entre sí en la 
Prueba I, cuyo texto presenta un mayor grado de especialización. Sistemáticamente, el nivel de formación académica se relaciona con los resultados, siendo el menor rendimiento el de los alumnos de primer semestre. En el texto II, que presentaba un menor grado de especialización, las tendencias se mantienen, pero con la diferencia que los alumnos de quinto y noveno semestre no se distinguen en su rendimiento.

\begin{tabular}{|c|c|c|c|c|}
\hline \multirow{2}{*}{$\begin{array}{c}\text { Experiencia } \\
\text { Académica }\end{array}$} & \multicolumn{2}{|c|}{ Prueba I } & \multicolumn{2}{|c|}{ Prueba II } \\
\hline Primero & 11,4 & c & 13,9 & b \\
\hline Tercero & 15,2 & b & 16,8 & a \\
\hline Quinto & 17,9 & a & 18,3 & a \\
\hline & \multicolumn{2}{|c|}{$<0,0001$} & $<0,0001$ & \\
\cline { 2 - 5 }
\end{tabular}

Tabla 2. Comparaciones entre los grupos para las pruebas I (texto 1) y prueba II (texto 2)

Letras distintas indican que las diferencias entre grupos fueron significativas.

Una interpretación preliminar de estos datos lleva a pensar que, cuando se trata de leer textos que requieren conocimiento específico de la disciplina y de alta especialización, las competencias requeridas deben ser suficientes para participar de la lectura y adquisición de conocimiento en el marco de la comunidad discursiva. Los estudiantes que se encuentran en una etapa inicial de su formación carecen de herramientas cognitivas y de la experiencia académica suficiente para resolver tareas de mayor complejidad. El avance en el proceso de formación académica y profesional capacita a los sujetos para las demandas intelectuales cada vez más exigentes. El hecho de que en la lectura del texto II, de carácter más bien didáctico, no se observen diferencias significativas entre alumnos de quinto y noveno semestre, debe ser interpretado como una evidencia de que, frente a tareas de menor especialización, la formación académica avanzada no constituye una variable determinante. La Figura 1 ilustra con mayor claridad las diferencias de rendimiento por cada subgrupo en ambas pruebas.

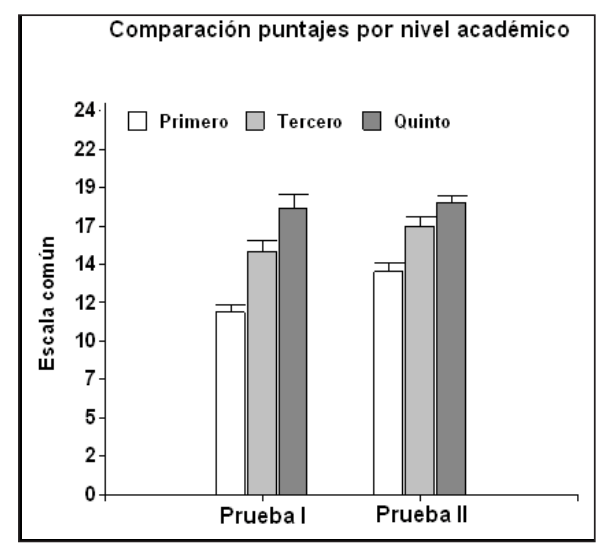

Figura 1. Comparaciones entre niveles académicos en ambas pruebas 


\subsection{RESULTADOS POR TIPO DE PREGUNTA}

En esta parte del artículo, se presentan los resultados por cada nivel de comprensión. Primero, los resultados del nivel global se resumen en la Tabla 3 y la Figura 2.

\begin{tabular}{|c|c|c|c|c|c|c|}
\hline Experiencia & & \multicolumn{2}{|c|}{ Puntaje Total Prueba I } & \multicolumn{2}{c|}{ Puntaje Total Prueba II } \\
\cline { 2 - 7 } Académica & $\mathrm{N}$ & Mín & Máx & & Mín & Máx \\
\hline Primero & 20 & 8 & 15 & & 10 & 20 \\
\hline Tercero & 20 & 8 & 21 & & 10 & 20 \\
\hline Quinto & 15 & 9 & 21 & & 15 & 21 \\
\hline
\end{tabular}

Tabla 3. Resumen de los puntajes de ambas pruebas aplicadas por experiencia académica

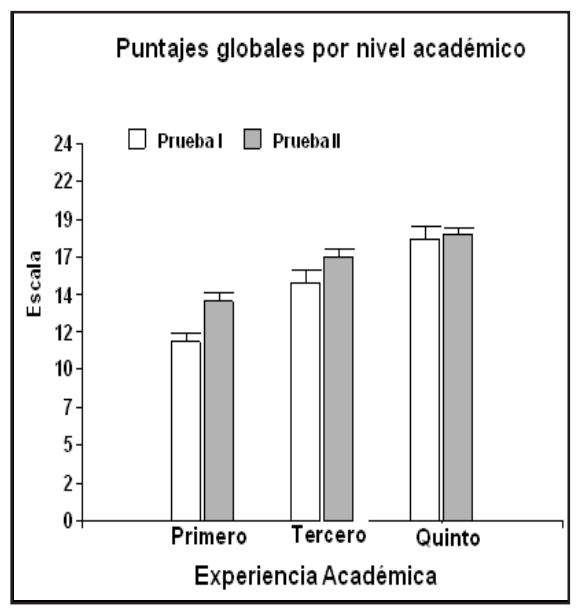

Figura 2. Puntajes globales por nivel académico en prueba I y II.

Estos datos muestran una nítida tendencia que vincula el nivel de formación y el grado de especialización textual con el rendimiento de los sujetos en las pruebas. Con toda claridad, los alumnos de primer y tercer año muestran diferencias en la lectura de ambos textos. Los estudiantes avanzados, sin embargo, pueden leer indistintamente ambos textos con igual facilidad/dificultad, lo que evidencia el peso de la formación académica.

Un análisis detallado del rendimiento por cada subtipo de pregunta, a su vez, permite confirmar con mayor fuerza la hipótesis de trabajo. La Tabla 4 presenta los resultados completos de estos datos con su correspondiente valor de significación estadística.

\begin{tabular}{|c|c|c|c|c|c|c|c|c|}
\hline Prueba & & Primero & & Tercero & & Quinto & & $\mathbf{P}$ \\
\hline & PRI & 6,2 & c & 8,7 & b & 11,4 & $\mathrm{a}$ & $<0.0001^{*}$ \\
\hline & PRE & 5,2 & $\mathrm{~b}$ & 6,6 & $a$ & 6,5 & $\mathrm{a}$ & $0.0005^{*}$ \\
\hline & FUNC & 0,9 & $\mathrm{~b}$ & 1,5 & $a$ & 2,2 & $\mathrm{a}$ & $0.0008^{*}$ \\
\hline
\end{tabular}




\begin{tabular}{|c|c|c|c|c|c|c|c|c|}
\hline \multirow{6}{*}{ I } & COND & 5,1 & $\mathrm{~b}$ & 7,1 & $\mathrm{a}$ & 7,7 & $\mathrm{a}$ & $<0.0001^{*}$ \\
\hline & IG & 0.95 & $b$ & 1,6 & $\mathrm{a}$ & 1,7 & $\mathrm{a}$ & $0.0037^{*}$ \\
\hline & PROP & 3,3 & b & 3,6 & $a b$ & 3,8 & $\mathrm{a}$ & $0.0430 *$ \\
\hline & LOC & 2,9 & $\mathrm{~b}$ & 4,7 & $\mathrm{a}$ & 5,5 & $a$ & $<0.0001 *$ \\
\hline & GLO & 4,0 & $\mathrm{~b}$ & 5,5 & $\mathrm{a}$ & 6 & $\mathrm{a}$ & $0.0004^{*}$ \\
\hline & ELAB & 1,3 & $b$ & 1,5 & $\mathrm{~b}$ & 2,6 & $\mathrm{a}$ & $0.0006^{*}$ \\
\hline \multirow{9}{*}{ II } & PRI & 7,3 & $\mathrm{~b}$ & 9,6 & $\mathrm{a}$ & 10,5 & $\mathrm{a}$ & $0.0001 *$ \\
\hline & PRE & 6,7 & $\mathrm{~b}$ & 7,1 & $a b$ & 7,8 & $\mathrm{a}$ & $0.0037^{*}$ \\
\hline & FUNC & 1,5 & b & 2,6 & $\mathrm{a}$ & 2,5 & $\mathrm{a}$ & $0.0003^{*}$ \\
\hline & COND & 6,9 & $\mathrm{~b}$ & 7,5 & $\mathrm{a}$ & 8,3 & $\mathrm{a}$ & $0.0053^{*}$ \\
\hline & IG & 1,2 & $\mathrm{a}$ & 1,3 & $\mathrm{a}$ & 1,5 & $\mathrm{a}$ & 0.1800 \\
\hline & PROP & 3,3 & b & 3,6 & $a b$ & 3,9 & $\mathrm{a}$ & $0.0197 *$ \\
\hline & LOC & 4,6 & $\mathrm{~b}$ & 5,6 & $\mathrm{a}$ & 6,0 & $\mathrm{a}$ & $0.0013^{*}$ \\
\hline & GLO & 5,0 & $b$ & 5,8 & $\mathrm{a}$ & 6,3 & $\mathrm{a}$ & $0.0037 *$ \\
\hline & ELAB & 1,2 & $\mathrm{~b}$ & 1,9 & $\mathrm{a}$ & 2 & $\mathrm{a}$ & $0.0039 *$ \\
\hline
\end{tabular}

Tabla 4. Comparaciones múltiples entre niveles académicos para Prueba I y Prueba II. Letras distintas indican que las diferencias entre grupos fueron significativas

A continuación, se presentan los resultados del nivel proposicional en la Figura 3. Estos datos muestran la misma tendencia observada en los otros niveles de procesamiento, a saber, que la formación académica tiene un peso determinante en la habilidad incluso para leer textos en un nivel de complejidad básico dentro de la estructura del texto como lo es el proposicional.

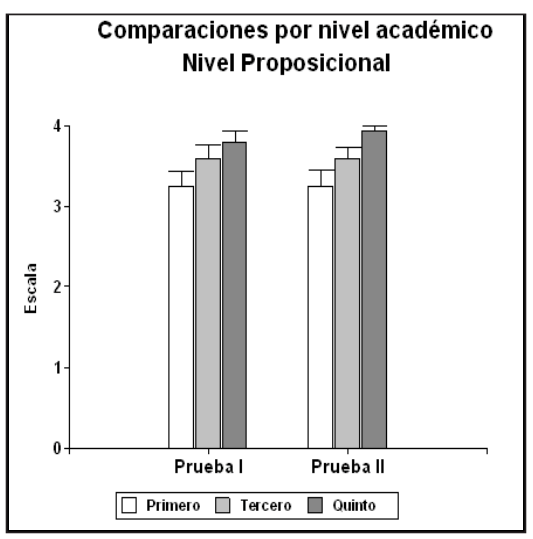

Figura 3. Comparaciones de rendimientos nivel Proposicional 
Continuando con el análisis, el nivel de procesamiento local, en el que se exige conectar dos o más proposiciones, mantiene lo observado en los casos anteriores, siendo más fuertes las diferencias en el caso de la Prueba 1, la que implica leer un texto de mayor demanda.

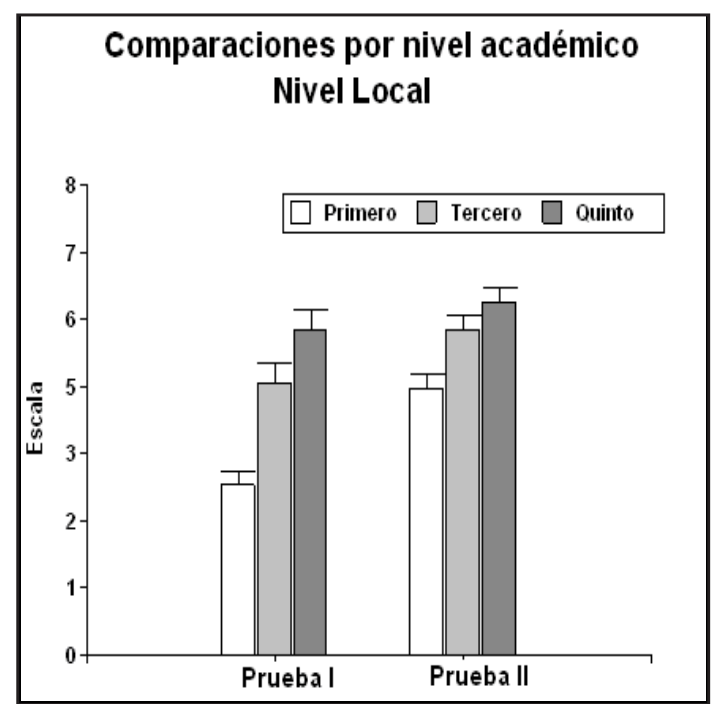

Figura 4. Comparaciones de rendimientos por nivel local.

En los pasos siguientes, se presentan los resultados del nivel más alto de procesamiento, así como del rendimiento en preguntas de respuesta explícita versus implícita y de diversos tipos de inferencias. Los datos no hacen más que confirmar lo encontrado hasta aquí.

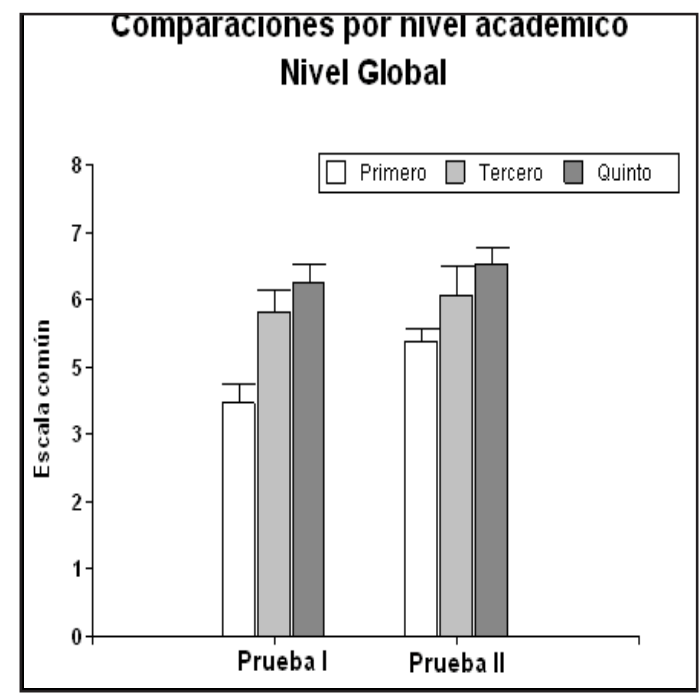

Figura 5. Comparaciones de rendimientos nivel global 


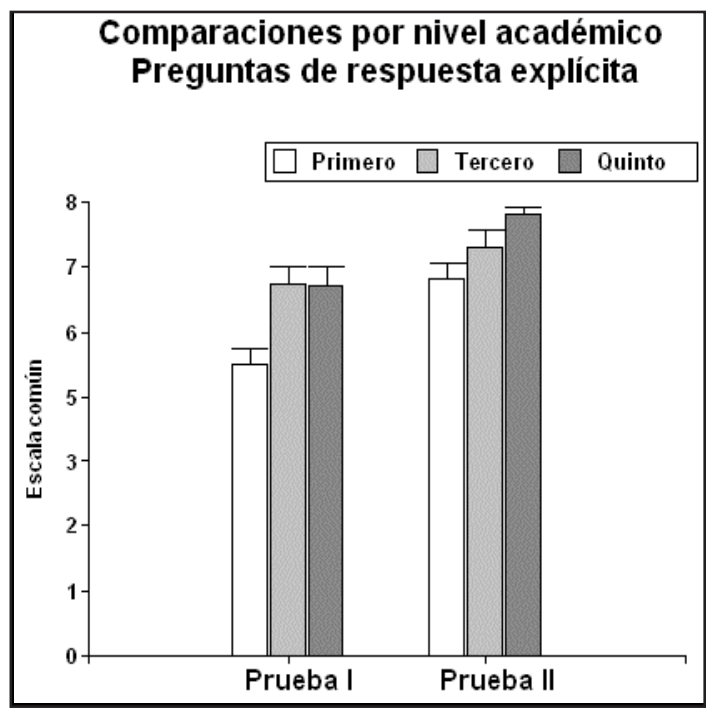

Figura 6. Comparaciones de rendimientos nivel PRE

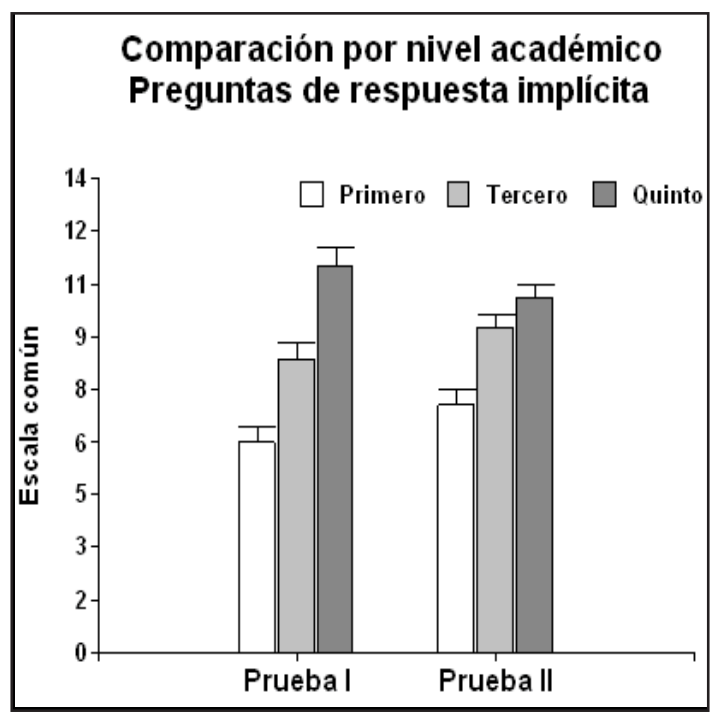

Figura 7. Comparaciones de rendimientos nivel PRI 


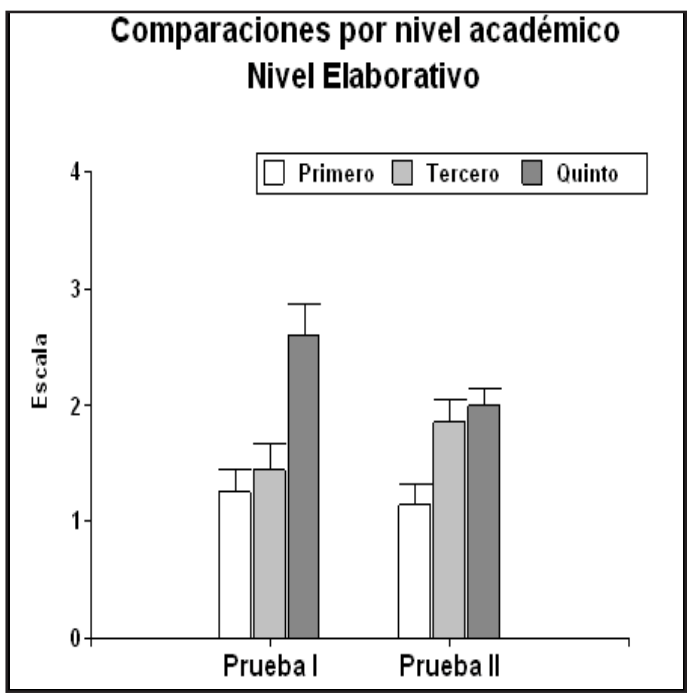

Figura 8. Comparaciones de rendimientos nivel Elaborativo

\section{CONCLUSIONES Y PROYECCIONES}

El propósito de la investigación que se informa aquí consistió en determinar si el nivel de formación académica y el grado de especialización del discurso inciden en el desempeño de los sujetos en tareas de comprensión de textos académicos específicos, en este caso, del área de las ciencias políticas. Consecuentemente, la hipótesis postulaba la existencia de esta relación entre las variables consideradas. Concluido el estudio, podemos confirmar que se ha comprobado la hipótesis de trabajo planteada y sostener que, efectivamente: 1) la experiencia académica, que supone un proceso de enseñanza-aprendizaje y sus resultados, y 2) las propiedades textuales del discurso académico, que permiten distinguir diversos grados de especialización, incidieron en el desempeño lector de los alumnos participantes en la investigación.

En relación con las líneas teóricas que abordan la comprensión de textos, es importante rescatar el hecho de que cada vez es más necesario tener en cuenta el desarrollo de modelos que integren factores cognitivos, lingüísticos y contextuales, con el fin de dar cuenta en mejor forma de un proceso complejo como es la comprensión lectora. En este sentido, se avizoran, a partir de la revisión de la literatura reciente, intentos integradores por parte de Cassany (2010), quien plantea que lo sociocultural sería el punto que tendería a integrar las dimensiones involucradas en el procesamiento de la información, que no sólo es individual sino que depende, como hemos visto a lo largo de este trabajo, de aspectos comunitarios y sociales. La noción de comunidad discursiva es ejemplo de aquello en el sentido de que en dicho contexto se restringe la integración por parte de nuevos individuos. La aprehensión de los géneros específicos de la comunidad también es factor a considerar, 
unido, inevitablemente, a la capacidad para procesarlos y comprenderlos en coherencia con el contexto pertinente en cada comunidad de práctica.

Por otra parte, el modelo utilizado en este trabajo para evaluar comprensión lectora en aspectos cuantitativos y cualitativos (Véliz \& Riffo, 1993) logró dar cuenta de las diferencias planteadas en las hipótesis de esta investigación. Esto demostraría que la propuesta es eficiente a la hora de medir también comprensión en textos especializados, puesto que está basada en principios fundamentales, rigurosamente ligados a bases teóricas del ámbito del procesamiento y representación del lenguaje. Se podría también tomar como base este modelo para diseñar uno que fuera específico para textos especializados, considerando aspectos característicos como las inferencias elaborativas y elementos de la cultura académica escrita (Cassany, 2010), entre otros factores, según la naturaleza humanista o científica de la comunidad en estudio. Tal desarrollo, sin embargo, va más allá de los límites del presente artículo.

\section{ANEXOS}

\section{Texto 1}

Uno de los rasgos esenciales de la operación de una democracia es la incertidumbre inevitable de sus resultados. En la sucesión indefinida de gobiernos electos, no hay garantía o determinismo alguno sobre la orientación programática o ideológica que ellos adopten, el contenido y naturaleza de las políticas que decidan e implementen, la calidad de su desempeño, cómo afecten los niveles de gobernancia existentes.

Puesto de otra manera, las reglas del juego constitutivas de una democracia y su operación no están asociadas necesariamente a ningún tipo específico de resultados. Ellas son compatibles con la elección de gobiernos de los más diversos signos: favorables a la globalización u opuestos a ella, adversarios de la desrregulación de mercados laborales o impulsores de ella, gobiernos religiosamente comprometidos y gobiernos que buscan profundizar procesos de secularización, y así por delante. En el extremo, gobiernos ideológicamente democráticos versus gobiernos que intentan destruir la democracia. La naturaleza de los resultados no está asociada a las reglas del juego democrático, sino a esa intersección entre la historia, sociología, cultura y economía política de la sociedad de que se trate, por una parte, con la política misma por la otra: el carácter de sus actores, sus intenciones, su inteligencia y talento, su humanidad.

Este rasgo básico de la democracia tiende frecuentemente a ser olvidado, o aun a ser sustituido por su premisa contraria: que la democracia está necesariamente asociada a un cierto tipo de resultados. Un buen ejemplo de ello se encuentra en la actual política estadounidense de promoción de la democracia, tal como la definió Bush hace cuatro años: "La política de los Estados Unidos consiste en buscar y apoyar el crecimiento de movimientos e instituciones democráticos en toda nación y cultura, con el objetivo último de acabar con la tiranía en nuestro planeta". Según la Secretaria de Estado Rice, esta política será exitosa no sólo por ser optimista e idealista, sino también porque descansa en premisas de sana lógica estratégica y en una comprensión adecuada de las nuevas realidades. De manera implícita, la apelación a una "sana lógica estratégica" connota la idea de una asociación o correlación entre reglas del juego democráticas y ciertos resultados específicos: 
fortalecimiento estatal, una sociedad más abierta, reformas económicas, políticas exteriores cuyo objetivo es la paz, gobernancia.

En armonía con esa política y su objetivo global, el gobierno estadounidense ha puesto en práctica un intervencionismo activo en la impulsión de procesos de democratización en países pertenecientes al mundo religioso y cultural islámico, obviamente a partir de la premisa de que esos procesos conducirán a resultados al menos razonablemente compatibles con el ideario político-cultural y económico que esa clase de resultados recién aludidos esbozan.

Hasta ahora, los sucesos parecen contradecir esa premisa. A la reciente victoria de Hamas en las elecciones palestinas cabría agregar el notable desempeño de la Fraternidad Musulmana en las elecciones parlamentarias en Egipto, la votación obtenida en Irak por una lista clerical chiíta vinculada a Irán, o la emergencia de Hezbollah en las elecciones del año pasado en Líbano.

Acotando los Riesgos: Quizás los primeros en identificar esa incertidumbre como problemática o riesgosa fueron teóricos de esa vertiente anti-política del liberalismo ejemplificada por figuras como Popper durante los años treinta del siglo pasado. La admisión a la lucha política de fuerzas antidemocráticas (fascistas o comunistas), populistas o partidarias de un intervencionismo estatal considerado nocivo para la economía de mercado, asociada a la posibilidad real de que esas fuerzas conquistaran el poder, ciertamente hacían de la incertidumbre una amenaza no menor. Para Popper la comprensión adecuada de la democracia es la que entrega Schumpeter: democracia como procedimiento, enfatizando el carácter políticamente competitivo de las elecciones, lo cual permite la adquisición y pérdida periódicas y pacíficas de poder, que es en definitiva lo que esencialmente entrega la democracia. Como es precisamente esa competición la que es responsable de la incertidumbre, proponerse como meta eliminarla virtualmente equivaldría a acabar con la democracia. Por consiguiente, el problema pasa a ser no su eliminación, sino acotarla. Inspiradas por ideas de ese cuño, los últimos cincuenta años han sido progresivamente pródigos en la identificación de mecanismos o artefactos institucionales cuyo sentido último es transformar la incertidumbre en incertidumbre acotada. Por ejemplo, la idea de que es legítimo que las normas constitucionales o legales prohíban la entrada a la lucha política de partidos o movimientos programáticamente orientados a destruir la democracia, o valores básicos constitutivos de ella contemporáneamente, continúa siendo controversial, pero prácticamente, de manera más o menos sutil, todo orden político democrático contemporáneo contiene barreras de entrada eficaces para partidos o movimientos de esa índole.

Mucho más visible, dramática y crecientemente menos polémica es la presencia en las democracias de hoy de un fenómeno cuya significado usualmente no se asocia a la finalidad de acotar la incertidumbre inherente a la democracia: la despolitización progresiva de más y más dominios de la vida. Ella consiste simplemente en dejar fuera del ámbito de las decisiones políticas sectores de actividad de diversa naturaleza, erigiendo barreras de entrada a la intervención política en ellos. La marcha triunfal de esta despolitización tiene lugar por dos carriles. Por una parte, actúa mediante lo que podría llamarse mercadización o privatización de actividades: la conversión de ámbitos públicos de actividades, por consiguiente sujetos a decisiones e intervenciones políticas, en ámbitos privados de actividades, sujetos fundamentalmente a lógicas de mercado y fuertemente protegidos de decisiones e intervenciones desde la política. Es la transformación en industrias de sectores muy diversos, y lo que permite hablar con propiedad de industria de la salud, de la educación, de la cultura, de la investigación científica, como también de los respectivos 
mercados que emergen en cada uno de esos dominios. Por otra parte, actúa a través de la tecnificación o tecnocratización de la política: el reemplazo o sustitución de lógicas políticas por tecnologías o saber cómo. Una expresión nítida de esta tecnificación es la reserva de ámbitos específicos de decisión a autoridades, cuya legitimidad descansa en la posesión y ejercicio de un saber especializado y sólo muy indirectamente en una confianza política otorgada por un Jefe de Ejecutivo o una decisión parlamentaria.

\section{Preguntas Prueba I}

1. Según el texto ¿A qué factores está asociada la naturaleza de los resultados en democracia? (Local - Condicional - PRE)

R. A la intersección de la historia, sociología, cultura y economía política por una parte. El carácter, intenciones inteligencia y talento de los actores de la política por otra.

2. ¿Cómo titularías el texto que acabas de leer? (Global - Idea Global - PRI)

R. La incertidumbre en la Democracia. (Respuesta que contenga los 2 conceptos relacionados)

3. ¿Según el párrafo 6, por qué se busca acotar la incertidumbre en democracia? (Local Condicional - PRI)

R. Porque hay una intención de ganar la competencia en las elecciones y mantener el poder, por parte de quienes gobiernan y tienen intenciones de seguir en el mando. Además, para evitar el riesgo de la entrada de sectores (como comunistas o fascistas) en la competición democrática.

4. ¿En qué consiste la política exterior de Estados Unidos? (Proposicional - PRE)

R. Buscar y apoyar el crecimiento de movimientos e instituciones democráticos en toda nación y cultura para terminar con la tiranía en el mundo.

5. ¿Cuál es la proporción entre democracia e incertidumbre? (Global Condicional - PRI)

R. A mayor incertidumbre, mayor democracia.

6. ¿En qué consiste la mercadización? (Proposicional - PRE)

R. En convertir actividades gubernamentales en ámbitos privados, sujetos a lógicas de mercado sin intervención política.

7. ¿Qué puedes concluir acerca de la relación entre incertidumbre y democracia? (Elaborativo - PRE)

R. Que no se pueden disociar, es decir, la incertidumbre pertenece a la democracia. Que la incertidumbre es necesaria para la democracia. Que en la democracia incluso se intenta mantener el poder acotándola. Los gobiernos tratan de controlar la incertidumbre para seguir en el poder, lo que no sería una perfecta democracia.

8. ¿Qué significa en el párrafo 3 el término "gobernancia"? (Local - Funcional - PRI)

R. El sentido es capacidad de crear condiciones para gobernar, lo que se asocia con un resultado favorable. 
9. De acuerdo con el texto ¿Qué sucede si la incertidumbre se elimina en el contexto de la democracia? (Global - Condicional - PRI)

R. Se acabaría la democracia y se estaría en un gobierno autoritario.

10. A partir de lo leído ¿Cómo se manifiesta la incertidumbre en Chile en los últimos años? (Elaborativo - PRI)

R. En las elecciones del 2009 se ha visto que ha habido pérdida de la concertación después de 20 años en el poder. Se ha acabado la incertidumbre al cabo de 20 años de Gobierno, ha habido una perdida periódica, lo que implica una autentica manifestación de la democracia. El nuevo Gobierno presenta incertidumbre, aquella que ya era escasa en la Concertación debido a lo acotada que la tenía.

11. ¿Cómo influye la despolitización en la democracia? (Global Condicional - PRE)

R. Influye en que la despolitización afecta a la democracia por ser un instrumento que acota la incertidumbre y por lo tanto, propicia que haya "menos" democracia en que los gobiernos no dan participación a los ciudadanos en las decisiones de los aparatos públicos.

12. ¿A qué se refiere el texto con la expresión "Este rasgo básico de la democracia" en la línea 1 del tercer párrafo? (Local - Funcional - PRI)

R. Que democracia no implica buenos resultados

\section{Texto 2}

El hombre, según lo hemos ya demostrado, nace con un título a la perfecta libertad y al disfrute ilimitado de todos los derechos y privilegios de la ley de la naturaleza. Tiene, pues, por naturaleza, al igual que cualquier otro hombre que haya en el mundo, poder no sólo para defender su propiedad, es decir, su vida, su libertad y sus bienes, contra los atropellos y ataques de los otros hombres, sino que tiene también poder para juzgar y castigar con la muerte cuando la atrocidad del crimen, en su opinión, así lo exige. Sin embargo, debido a que una sociedad política no puede existir ni subsistir si no posee en sí misma poder para defender la propiedad, y, por tanto, para castigar las faltas de los miembros de esa sociedad, resulta que una sociedad política únicamente puede existir allí, y sólo allí, donde cada uno de los miembros ha renunciado a su poder natural poniéndolo en manos de la comunidad en todos aquellos casos en que puede recurrir en demanda de protección a la ley establecida por esa sociedad. Así, al quedar excluido el juicio particular de cada uno de los miembros, la comunidad se convierte en árbitro mediante el establecimiento de reglas permanentes, imparciales e iguales para todas las partes; y, por intermedio de hombres autorizados por la comunidad para la ejecución de esas normas, resuelve todas las diferencias que puedan surgir entre los miembros de esa sociedad en cualquier asunto de derecho, y castiga los delitos que cualquier miembro haya cometido contra la sociedad, aplicando las penas que la ley establece. De ese modo resulta fácil discernir quiénes están reunidos en sociedad política y quiénes no. Aquellos que se encuentran unidos formando un mismo cuerpo, y que poseen una ley común sancionada y un organismo judicial al cual recurrir, con autoridad para resolver las disputas entre ellos y castigar a los culpables, viven en sociedad civil los unos con los otros; empero, aquellos que no disponen de una instancia de apelación común, quiero decir, de una instancia de apelación en la tierra, aún 
permanecen en estado de naturaleza, y cada uno de ellos, a falta de otro juez, es juez y ejecutor por sí solo, lo que constituye como lo he manifestado anteriormente, el estado de naturaleza perfecto. (VII, 87).

El Estado, de esa manera, viene a disponer del poder para establecer qué castigo habrá de aplicarse a las diferentes transgresiones que considera merecen una sanción, cometidas por los miembros de la sociedad (este es el poder de hacer leyes); así como tiene el poder de castigar cualquier daño infligido a uno de sus miembros por alguien que no lo es (es decir, el poder de la guerra y de la paz). Y el objeto de esos poderes no es otro que la defensa de la propiedad de todos los miembros de esa sociedad, hasta donde sea posible. Pero aunque cada hombre que entra en sociedad renuncia a su poder de castigar, de acuerdo a su particular y propio juicio, los atropellos contra la ley de la Naturaleza, resulta que por el hecho mismo de haber entregado a la legislatura el poder de juzgar las ofensas, en todos aquellos casos en que se puede apelar al magistrado, ha puesto también su fuerza a disposición del Estado, concediéndole el derecho de emplearla cada vez que fuere necesario, para la ejecución de las sentencias dictadas por la comunidad; sentencias que, en efecto, son sus propios juicios, pues son dictadas por él mismo o por su representante. Allí se encuentra el origen del poder legislativo y del poder ejecutivo de la sociedad civil, a saber, el poder de juzgar, conforme a leyes establecidas, en qué grado se han de castigar las ofensas cuando éstas se cometen dentro del Estado; así como allí radica el poder de juzgar en determinadas ocasiones, sobre la base de las circunstancias presentes del hecho, en qué grado se han de vindicar los daños cometidos desde el exterior. En ambos casos, cuando ello es necesario, la sociedad civil puede emplear la fuerza de todos sus miembros. (VII, 88).

Por consiguiente, siempre que un número de hombres se une en sociedad renunciando cada uno de ellos a su poder para ejecutar la ley de la naturaleza, cediéndoselo a la comunidad, allí, y sólo allí, existe una sociedad civil o política. Y esto ocurre siempre que cierto número de hombres que vivían en el estado de naturaleza se unen en sociedad para formar un pueblo, un cuerpo político, bajo un gobierno supremo; o cuando alguien se asocia e incorpora a un gobierno ya establecido. Pues, con ello, un hombre autoriza a la sociedad o, lo que es lo mismo, a su poder legislativo, para hacer leyes en su nombre, conforme lo exija el bien público de la sociedad, y para ejecutarlas cuando se necesite su ayuda (como sí se tratase de sus propias resoluciones).

Eso es lo que saca a los hombres del estado de naturaleza y los coloca dentro de la sociedad civil, es decir, el hecho de establecer un juez en la tierra con autoridad para resolver todas las controversias y reparar los daños que pueda sufrir cualquiera de los miembros de esa sociedad. Ese juez es el poder legislativo, o lo son los magistrados que él mismo designe. Siempre que encontremos un cierto número de hombres que no obstante hallarse asociados entre sí no dispongan de ese poder decisivo al cual apelar, podemos decir que ellos permanecen viviendo en el estado de naturaleza. (VII, 89).

\section{Preguntas Prueba II}

1. ¿Quiénes instituyen un estado político? (Proposicional- PRE)

R. Los propios hombres al reunirse en sociedad que renuncian a sus derechos naturales para traspasarlos a la sociedad. 
2. Dado que en un estado político los hombres ceden sus derechos para que alguien los represente ¿Por qué existen delitos en este estado? (Elaborativo - PRI)

R. Porque hay ciudadanos que no tienen propiedades y pretender forzosamente tener algunas y recurren a la sustracción de las propiedades de los demás hombres, lo que implica también que el estado natural se hace presente en la conducta de los hombres aun estando en sociedad.

3. ¿Por qué debe existir entre los hombres una sociedad política? (Local Condicional PRI) R. Porque así se permite una vida regulada y que garantiza seguridad para los hombres constituidos en sociedad.

4. ¿En la lógica que presenta el texto, qué pasaría si una persona de la comunidad no quiere respetar las normas o reglas de la sociedad? (Elaborativo - PRI)

R. Tendría que dejar la sociedad en la que está viviendo, perdiendo todos los derechos de bienes y protección asociados a su adscripción en tal sociedad. Además si infringe las normas sería castigado.

5. ¿Qué significa la expresión "de esa manera” en el párrafo 5? (Local - Funcional - PRI) $\mathrm{R}$. El hecho de representar a los miembros en sociedad política

6. De acuerdo con el párrafo 1 ¿con qué nace el hombre? (Proposicional - PRE)

R. Nace con un título a la perfecta libertad y al disfrute ilimitado de todos los derechos y privilegios de la ley de la naturaleza

7. ¿Qué poderes posee el hombre en estado de naturaleza perfecta? (Local - Condicional - PRE)

R. El poder de juzgar bajo su propio criterio y de administrarse se acuerdo con su juicio personal. Derecho a defender su propiedad, sus bienes, a juzgar a partir de su criterio e impartir sentencias.

8. Indique en una línea la idea que da cuenta de la temática del texto (Global - Idea Global - PRI)

R. El gobierno está constituido por instancias que garantizan los derechos naturales que los hombres han depositado en él.

9. ¿A qué se refiere el texto con el término "estado natural”? (Local - Funcional - PRI)

R. Estado de predisposición humana para la supervivencia.

10. ¿Qué consecuencias tiene para el hombre el hecho de renunciar a sus derechos en estado natural? (Global - Condicional - PRI)

R. No poder defenderse y juzgar por sí solo, no tener reglas o normas particulares, ceder la representación de sí mismo ante otro que lo haga ante instancias externas.

11. ¿Según el texto ¿Por qué los hombres no pueden vivir en estado natural si quieren estar juntos? (Global - Condicional - PRI) 
R. Porque habría peligro de muerte para cada uno de ellos. Es un requisito para vivir en sociedad.

12. ¿Por qué existe en el estado político un poder legislativo? (Global - Condicional - PRE) R. Existe porque se necesita establecer normas de convivencia entre los miembros para evitar atropellos a partir de las diferencias que puedan surgir entre los miembros de la sociedad.

\section{REFERENCIAS BIBLIOGRÁFICAS}

Carlino, P. (2005). Escribir, leer y aprender en la Universidad. Buenos Aires: Fondo de Cultura Económica

Cassany, D. (2008). Metodología para trabajar con géneros discursivos. Espezialitateko Hizkerak eta Terminologia III, 9-24. Argitalpen zerbitzua.

Cassany, D. (2010). Sobre las interferencias en ELE y L2. Revista Nebrija de Lingüística Aplicada, 7, 4, 32-43.

Gernsbacher, M. A. (1997). Two Decades of Structure Building. Discourse Processes, 23, 265-304.

Johnson-Laird, Ph. (1983). Mental Models. Towards a Cognitive Science of Language, Inference, and Consciousness. Cambridge: Harvard University Press.

Kintsch, W. (1998). Comprehension: A Paradigm for Cognition. New York: Cambridge University Press.

Parodi, G. (2005). La comprensión del discurso especializado en ámbitos técnico profesionales: Aprendiendo a partir del texto. Revista Signos, Vol. 38, N ${ }^{\circ}$ 58, 221-267.

Parodi, G. (2008). Géneros Académicos y Géneros Profesionales: Accesos Discursivos para Saber y Hacer. Valparaíso: Ediciones Universitarias de Valparaíso.

Van Dijk, T. \& Kintsch, W. (1983). Strategies of Discourse Comprehension. New York: Academic Press.

Véliz, M. \& Riffo, B. (1993). Comprensión textual: criterios para su evaluación. RLA, Vol. 31, 163-190.

Zwaan, R. \& Radvansky, G. (1998). Situation Models in Language Comprehension and Memory. Psychological Bulletin, 123, 162-185. 